\title{
People's willingness to accept overdetection in cancer screening: population survey
}

\author{
(ब) $(\mathbb{Q} \Theta$ OPEN ACCESS
}

\author{
Ann Van den Bruel senior clinical research fellow ${ }^{1}$, Caroline Jones senior researcher ${ }^{1}$, Yaling Yang \\ senior researcher in health economics ${ }^{1}$, Jason Oke medical statistician ${ }^{1}$, Paul Hewitson senior \\ researcher ${ }^{2}$
}

'Nuffield Department of Primary Care Health Sciences, Radcliffe Observatory Quarter, University of Oxford, Oxford OX2 6GG, UK; ${ }^{2}$ Nuffield Department of Population Health, Richard Doll Building, University of Oxford, Oxford OX3 7LF, UK

\begin{abstract}
Objectives To describe the level of overdetection people would find acceptable in screening for breast, prostate, and bowel cancer and whether acceptability is influenced by the magnitude of the benefit from screening and the cancer specific harms from overdetection.

Design Online survey. Women were presented with scenarios on breast and bowel cancer, men with scenarios on prostate and bowel cancer For each particular cancer, we presented epidemiological information and described the treatment and its consequences. Secondly, we presented two different scenarios of benefit: one indicating a 10\% reduction in cancer specific mortality and the second indicating a $50 \%$ reduction.

Setting Online survey of the population in the United Kingdom.

Participants Respondents were part of an existing panel of people who volunteer for online research and were invited by email or online marketing. We recruited 1000 respondents, representative for age and sex for the UK population.

Main outcome measures Number of cases of overdetection people were willing to accept, ranging from 0-1000 (complete screened population) for each cancer modality and each scenario of benefit.

Results There was large variability between respondents in the level of overdetection they would find acceptable, with medians ranging from 113 to 313 cases of overdetection per 1000 people screened. Across all scenarios, $4-7 \%$ of respondents indicated they would accept no overdetection at all compared with $7-14 \%$ who thought that it would be acceptable for the entire screened population to be overdetected. Acceptability in screening for bowel cancer was significantly lower than for breast and prostate cancer. People aged 50 or over accepted significantly less overdetection, whereas people with higher education levels accepted more; $29 \%$ of respondents had heard of overdetection before.
\end{abstract}

Conclusions Acceptability of overdetection in cancer screening is variable. Invitations for screening should include clear information on the likelihood and consequences of overdetection to allow people to make an informed choice.

\section{Introduction}

In cancer screening, overdetection is defined as the detection of a cancerous lesion through screening that would otherwise not have caused any symptoms or early death. ${ }^{1}$ It is considered the most harmful effect of cancer screening as it unnecessarily labels people as "cancer patients," leads to treatments causing harm but no benefit, and diverts healthcare activity from patients who genuinely need it. ${ }^{2}$

Specifically in cancer screening, the main drivers of overdetection are the cancer's speed of progression and lead time. Some cancer lesions progress slowly or not at all; as a result they will not cause symptoms in the affected person's lifetime. ${ }^{1}$ Secondly, screening will detect cancer before it causes symptoms, advancing the moment of diagnosis, which is called lead time. Especially in the presence of competing risks because of other health conditions or advanced age, people might die before the cancer would have become symptomatic, making the earlier detection of the cancer futile. The risk of overdetection is particularly large in the detection of small cancer lesions: their progression is uncertain, and, if they do progress, lead time might be substantial.

In addition to harms from being labelled as patient with a cancer, ${ }^{34}$ overdetection is harmful for the individual because it leads to unnecessary testing and treatment. Overdetection in breast cancer screening results in more breast surgery including mastectomy ${ }^{5}{ }^{6}$ and deaths from cardiovascular disease and secondary cancers after radiotherapy. ${ }^{7}$ In screening for prostate 
cancer, harms from overdetection include urinary incontinence and erectile dysfunction after prostatectomy and bowel dysfunction after radiotherapy. ${ }^{8}$ Screening for bowel cancer is associated with the risks of further testing with colonoscopy and the associated complications of this procedure (bleeding and perforation). ${ }^{9}$

Because cancer screening can lead to benefit in some people and harm in others, there is general consensus that people should be allowed to make an informed choice before deciding to participate. Patient information leaflets, however, seldom include specific information on overdetection. ${ }^{10-12}$ This might be because of uncertainty on the extent of overdetection, the difficulty in explaining the concept in lay terms, or fears it could lower participation..$^{13}$ As a result, overdetection is largely unknown to most people, ${ }^{14}$ and many are surprised when they are informed about its possibility. ${ }^{15}$ In addition, we know little about how much overdetection people would consider acceptable, which could depend on the benefit that could result from screening or the consequences of overdetection, which are different according to which cancer is being overdetected.

We examined how much overdetection people would accept when participating in screening for breast cancer, prostate cancer, and bowel cancer and whether acceptability is influenced by the magnitude of the benefit from screening and the cancer specific harms from overdetection

\section{Methods}

We performed an online survey of people aged 18 or older living in the United Kingdom. Potential respondents were part of an existing panel of people who volunteer for online research and were invited by email or online marketing in August 2014. Each respondent received a financial incentive of about $£ 1$ ( $€ 1.4$, $\$ 1.5)$ to participate. The sample was selected to be representative for age and sex according to the 2011 UK census, and sample size was based on the achievement of a $95 \%$ confidence interval of $<20$ around a hypothesised mean of 20 cases of overdetection that would be acceptable for bowel cancer screening (requiring at least 987 respondents).

The survey included questions on three different types of cancer screening: breast cancer (for women), prostate cancer (for men), and bowel cancer (for both men and women). For each type separately, we presented the absolute number of cases per year in the UK and a description of the treatment, including its adverse effects. Incidence estimates and evidence on treatment and adverse effects were drawn from the respective Cochrane Systematic Reviews, ${ }^{5-16}$ relevant National Institute for Health and Care Excellence (NICE) guidance on diagnosis and treatment of early and locally advanced breast cancer (CG80), prostate cancer (CG175), and colorectal cancer (CG131), the UK Screening Portal (www.screening.nhs.uk), the National Bowel Cancer Audit report, ${ }^{17}$ and the Second All Breast Cancer Report. ${ }^{18}$

Immediately after we presented the background information for that particular type of screening, we presented two hypothetical screening scenarios. Both scenarios described a population of 1000 respondents, with a $5 \%$ incidence of cancer and $1 \%$ cancer specific mortality. The first scenario represented a $10 \%$ relative reduction of cancer specific mortality as a result of screening; the second scenario a $50 \%$ relative reduction. All information was presented in written format with absolute numbers and illustrated graphically to increase understanding ${ }^{19}$ (see appendix figure S1). We kept the incidence, cancer specific mortality, and benefit constant over the three types of screening, the only difference between them was the treatment and its consequences (appendix table S1).

We consistently used the term overdetection rather than overdiagnosis to avoid confusion with misdiagnosis as documented in previous studies. ${ }^{15}$ Immediately after each scenario of benefit, we asked respondents about the maximum number of people overdetected and overtreated that they would accept for the given benefit. The respondents were allowed to indicate a number between 0 (the minimum) and 1000 (entire population as maximum). At the end of the survey respondents were asked "Do you have anything else to add about overdetection in cancer screening?"

The survey was piloted in a small group of the general public: face-to-face cognitive interviews were conducted with three members of our patient and public involvement panel to ascertain the comprehensibility of items and visual presentations, after we amended the survey to increase comprehensibility.

\section{Analyses}

Data on respondents' baseline characteristics including age, sex, previous diagnosis of cancer, any chronic conditions, education level of degree or above, knowledge of overdetection, and previous participation in cancer screening are reported descriptively including $95 \%$ confidence intervals. Acceptability of overdetection for each scenario of each screening modality was not normally distributed. As a result, we report summary statistics using medians and $95 \%$ confidence intervals. We used non-parametric bootstrap to approximate the sampling distribution of median acceptability of overdetection and derived 95\% confidence intervals using the centile method. Distributions were smoothed with an Epanechnikov kernel function (figure $\downarrow$ ). We tested whether there were significant differences in the median acceptability of overdetection across different types of screening using the Wilcoxon test for paired data. In addition using the Mann-Whitney test to examine whether median acceptability was associated with respondents' baseline characteristics for both scenarios on bowel cancer. For all univariate analyses, we categorised age in those aged 50 or older versus younger than 50 because most cancer screening programmes start after the age of 50 .

Secondly, we performed a logistic regression analysis to explore whether respondents' baseline characteristics were associated with either very high or very low acceptance levels of overdetection. Very high acceptance of overdetection was defined as accepting $\geq 800$ cases of overdetection for any scenario of any of the types of screening based on the distributions that showed a marked increase after 800 cases, corresponding with the 80-88th centiles across scenarios. The threshold for very low acceptance was set accordingly at the 20th centile, corresponding with a maximum of 30 cases of overdetection. Variables were considered to be significant in the regression analyses at $\mathrm{P} \leq 0.05$. We initially fitted a logistic regression model with age as a continuous covariate, but after checking the model it was clear that age did not have a linear relation with either very high or very low acceptance of overdetection and so we categorised age as $<50$ and $\geq 50$.

All quantitative analyses were performed in Stata v.11.2.

We examined and compared the free text responses of respondents accepting very high ( $\geq 800$ cases) or very low $(\leq 30)$ levels of overdetection for at least one scenario. We conducted thematic analysis based on emergent themes in the data. Coding schemes were produced based on the content of the free text comments, and the data were coded accordingly. Separate coding schemes were produced for respondents with very high and very 
low levels of overdetection, and the data within and between the two groups were compared.

\section{Results}

The survey was completed by 1000 respondents (234 people started the survey but did not finish, which corresponds to a $19 \%$ attrition rate). Table 1 shows the baseline characteristics $\Downarrow$. Compared with the 2011 census, the sample was less ethnically diverse (UK population $85 \%$ white $v 91 \%$ in our sample) and more highly educated (UK population $27 \%$ degree level or above $v 43 \%$ in our sample) but representative for age and sex.

Slightly less than $10 \%$ of respondents had been diagnosed with cancer before, of which breast cancer was the most common $(n=23)$ followed by skin $(n=17)$ and prostate cancer $(n=8)$. About half of respondents $(54 \%)$ had participated in cancer screening at least once in their lifetime. Participation was higher for women $(73 \%)$ than for men (34\%): $42 \%$ of all women reported having participated in breast cancer screening, $58 \%$ in cervical cancer screening, and $25 \%$ in bowel cancer screening; $34 \%$ of all men reported having participated in bowel cancer screening and $11 \%$ had had a prostate specific antigen test for prostate cancer. Restriction of these results to respondents who could have been invited based on age (cervical cancer from age 25, breast cancer from age 50, bowel cancer from age 60), participation was $82.0 \%$ for cervical cancer (164/200), $64.2 \%$ for breast cancer $(291 / 453)$ and $75.5 \%$ for bowel cancer in women (80/106), and 75.6\% for bowel cancer in men (124/164). We have not presented estimates for prostate cancer as there is no screening programme in the UK.

Less than a third of respondents (29.2\%) said they had heard about overdetection before; in respondents aged 50 or older, a similar proportion reported knowledge of overdetection (29.7\%). The most common source of information on overdetection was the media (44\%), followed by doctors ( $28 \%$, of whom half were general practitioners), and family and friends (19\%). Only two respondents said they had read about overdetection in the leaflet that came with a screening invitation.

\section{Overall acceptability}

The distributions of each scenario for each type of screening show that acceptability peaks at around 100 cases, followed by a long right tail with a moderate increase around 500 and a more pronounced increase between 800-1000 (figure $\downarrow$ ).

There were significantly higher acceptance rates for the $50 \%$ reduction of cancer specific mortality compared with the $10 \%$ reduction for all screening modalities (all $\mathrm{P}<0.001$ ), although acceptability of overdetection did not increase fivefold when benefit increased fivefold (table $2 \Downarrow$ ). Women accepted significantly more overdetection in breast cancer than in bowel cancer (medians 150 and 120 for scenario $1, \mathrm{P}<0.001$; and 314 and 231 for scenario 2, $\mathrm{P}<0.001$ ), and, similarly, men accepted more overdetection in prostate cancer than in bowel cancer (medians 126 and 112 for scenario $1, \mathrm{P}=0.006$; and 231 and 199 for scenario $2, \mathrm{P}<0.001$ ).

Across all scenarios, $3.5-7.4 \%$ of people indicated they would not accept any level of overdetection, compared with 7.1-13.9\% of people who would accept the entire population of 1000 people to be overdetected to avoid one or five people dying from cancer (table $2 \Downarrow$ ).

We did not find a significant difference between men and women in the level of overdetection they would accept in screening for bowel cancer (table $3 \Downarrow$ ). Additionally, we found no significant association with a previous diagnosis of cancer, having a long term condition, or knowledge of overdetection on acceptability of overdetection. People aged 50 or older, however, accepted significantly less overdetection than younger respondents in both scenarios. Respondents with a degree or above accepted significantly more overdetection than respondents with lower levels of education, although this was apparent only in the scenario with higher benefit.

\section{Very low and very high acceptance levels}

A total of 235 respondents indicated they would accept 800 cases of overdetection or more on at least one scenario for any type of screening. Only the presence of a chronic condition was associated with higher acceptance levels: odds ratio 1.55 (95\% confidence interval 1.14 to 2.14 ). Of those 235 respondents, 160 entered a free text comment. We removed nonsensical $(n=5)$ and "no comment" or similar $(\mathrm{n}=72)$ entries, leaving 83 comments for analysis. The most salient theme was that it is better to save one life, even if there are negative consequences, and that overdetection is better than cancer not being detected. Some of the comments revealed that personal experiences, age, and the type of cancer treatment influenced their attitude. Respondents expressed surprise about overdetection and thought that screening and cancer treatment needed to be improved, including better screening, more funding, wider availability of screening, fewer side effects from treatment, and discussion of options after a positive screening result (quotes provided in appendix table $2 \mathrm{~S}$ ).

In contrast, 340 respondents indicated that they would accept 30 cases of overdetection or fewer on at least one scenario for any type of screening. Being aged 50 or older was significantly associated with such low acceptance levels: odds ratio 1.93 (95\% confidence interval 1.43 to 2.61 ). On the other hand, respondents who had participated in cancer screening at least once were less likely to be in this low acceptance category: 0.68 ( 0.49 to 0.94 ). Of those 340 respondents, 230 entered a free text comment; after removal of "no comment" or similar entries $(n=113)$, we analysed 117 comments. In contrast with the high acceptance group, respondents with low acceptance thought that the negative consequences of overdetection outweigh the benefit of saving a small number of lives and that it is important to prevent unnecessary deaths linked to overdetection. Similarly to the high acceptance group, respondents expressed surprise, and sometimes concern, about overdetection, and thought that more should be done to inform the public and that improvements are needed to screening and treatment. It was also suggested that the public should be better educated about symptoms as an alternative to screening.

\section{Discussion}

\section{Principal findings}

Levels of acceptance of overdetection in cancer screening vary widely, ranging from zero to acceptance in the complete population. In this survey of a sample of the general UK population we found very high median acceptance levels for each cancer studied and for every hypothetical benefit.

Strikingly, more people would accept overdetection in the entire population than the number who would accept no overdetection at all. We found no difference between men and women in their acceptability of overdetection in screening for bowel cancer, although both men and women accepted less overdetection in bowel cancer than in prostate and breast cancer screening, respectively. People aged 50 or over accepted less overdetection, whereas respondents with a degree or above accepted more (although the latter only in the higher benefit scenario, 
suggesting that they made a more explicit trade off between benefit and risk). People who had a chronic condition were more likely to accept overdetection in $\geq 80 \%$ of the population. As the benefit increased fivefold, the number of people in whom survey respondents thought overdetection was acceptable also increased, although not by fivefold.

As only a minority of respondents had previously heard about overdetection, questions can be raised as to whether respondents had truly grasped the meaning and consequences of overdetection, especially because the concept of overdetection is complex and there is a limit to how information can be conveyed without a face-to-face contact. In that respect, it is possible that acceptance levels for bowel cancer were significantly lower because of a learning effect during the survey, as bowel cancer was the second screening type presented to each respondent. The description of bowel cancer, however, also included specific information on mortality risk associated with surgery for bowel cancer, which could also have influenced acceptance levels, as suggested by free text entries from respondents with very high acceptance of overdetection. Although there is a real possibility that some respondents did not understand the information presented, the fact that acceptability of overdetection increased as benefit increased suggests that on the whole respondents made a trade-off between benefits and harms. This assumption is further reinforced by the free text comments, which suggest some people would prefer to experience harm from cancer treatment rather than from cancer itself.

Strikingly, only two people said they had seen information on overdetection in a screening leaflet, although $56 \%$ of respondents had been invited for cancer screening at least once in their lifetime. This was also confirmed by the free text analyses, which showed that several respondents were surprised at the existence of overdiagnosis and thought that the public should be better informed.

\section{Strengths and limitations}

Our survey was performed in a large sample of the UK population, representative for age and sex and not restricted to people who are eligible for screening or without a history of cancer. Before asking respondents to indicate their acceptability of overdetection, we presented cancer specific information on the consequences of overdetection to allow them to make an informed choice. We acknowledge that the way information was conveyed might influence the findings, which is why we opted for a combination of written and graphic information to maximise comprehensibility.

Despite the existence of several high quality systematic reviews collating all the available evidence on benefits and harms of screening, we found it particularly difficult to obtain reliable estimates of the harms from overdetection and consequently the descriptions still lacked specific data. As a result, we were unable to present head-to-head comparisons-that is, the number of deaths caused by treatment of overdetetected cancer versus the number of deaths from cancer avoided by screening.

We did not present the different types of screening in random order, making bowel cancer the second type presented to each respondent. This could have had an impact on the acceptability levels reported for bowel cancer, as most respondents had not heard of overdetection before and there might have been a learning effect after the first screening type. For the same reason, it is possible that respondents might not have fully understood the questions. We tested the survey face to face in a small group, however, which showed that the questions, although perceived as "not easy to answer," were understood by our test panel and similar high ranges of acceptability were obtained during the test. The free text comments also revealed a depth of understanding and consideration of the questions.

\section{Comparison with existing literature}

An earlier online survey on acceptability of overdetection found a median of one case of overdetection for one person to be saved. ${ }^{20}$ This is different from the 113-150 cases of overdetection found acceptable in our study. This large discrepancy could be because the earlier survey limited the responses to 100 people overdetected from 1000 screened people and split possibilities over seven categories, which were skewed towards low acceptability. In contrast, we allowed any estimate ranging from 0 up to the total population of 1000 screened people.

Our finding that a proportion of respondents would accept very high levels of overdetection is similar to other related work that seems to suggest some people are willing to tolerate high rates of false positive diagnoses with computed tomography colonography in exchange for the diagnosis of an extracolonic malignancy, with more than $20 \%$ of respondents accepting invasive testing even when this resulted in a risk of death that was equivalent to the chance of finding an extracolonic malignancy. ${ }^{21}$ On the other hand, informing people about the risks associated with cancer screening has been shown to decrease participation, ${ }^{22}$ and presenting people with risk information (one death per 10000 colonoscopies evoked negative emotional responses. ${ }^{23}$ This again seems to support the finding that people might respond differently to potential overdetection and risks associated with cancer screening.

\section{Implications for clinicians and policy makers}

Lack of knowledge on overdetection seriously hampers informed decision making in participation in cancer screening. In the past decade, few improvements were seen in how people are informed by leaflets. ${ }^{12} 24$ In general, the potential benefit is more commonly presented than the harms, and the effects are imprecise and presented in relative risks. ${ }^{25}$

Some countries attempt to inform potential participants in screening about overdetection, but the information is seldom specific enough to make a truly informed choice. The current NHS leaflet for breast cancer screening indicates that a woman is three times more likely to experience overdetection than to avoid death from breast cancer by participating in the screening programme. Although there is debate about the accuracy of this estimate, ${ }^{12}$ the leaflet currently does not include specific risks associated with overdetection other than receiving unnecessary treatment. Moreover, reduction in mortality from breast cancer was the most common information provided in 47 Italian leaflets, but none included any information on risks or potential harms. ${ }^{10}$ The NHS leaflet for bowel scope screening includes no information on possible overdetection (July 2013) nor does the NHS Bowel Cancer Screening website (www. cancerscreening.nhs.uk/bowel). Similarly, a third of 41 information materials used to inform people about bowel cancer screening in Germany did not provide any information on harms. ${ }^{26}$ Although the trials on bowel cancer screening did not show any increase in cancer diagnoses in the screened population because precancerous lesions (polyps, adenomas), which are targeted for treatment during colonoscopy, were not included in the cancer outcome, the Minnesota trial on bowel cancer screening showed that $47 \%$ of respondents received between one and six colonoscopies in the first 15 years. ${ }^{9}$ 
One could argue that it is impossible to convey all important information in one leaflet. General awareness about overdetection could be improved by mass media campaigns. Such campaigns have the additional benefit of reaching both the general public and clinicians, as evidence seems to suggest that clinicians have an equally poor understanding of diagnostic accuracy. ${ }^{27}$ In the presence of an ongoing education campaign, leaflets with invitations for cancer screening could then be more explicit about the likelihood and consequences of overdetection for that particular screening.

\section{Unanswered questions and future research}

Longitudinal studies could provide more insight into whether people's individual acceptability varies on the basis of personal experiences, media coverage of high profile patients with cancer, etc. In addition, despite our best efforts, we were not able to recruit a sample that was representative for UK education level and ethnicity, which means our findings might not be generalisable to populations with a more diverse background. The current lack of information might be symptomatic of an underlying evidence void on the exact consequences of overdetection. In addition, most people did not have any recollection of overdetection being mentioned in leaflets when they were invited to attend screening. To allow people to make an informed choice that is aligned with their personal values, we should get better at measuring not only benefits but also the harms related to screening. As a research community, it is imperative we develop more precise ways of communicating the benefits and risks of screening and furthermore, measuring the effectiveness of presenting this information to enable people to make informed choices about their screening decisions.

\section{Conclusion}

People have highly variable views on how much overdetection is acceptable in cancer screening. They should therefore be informed about the risk of overdetection and its consequences before deciding to participate. To enable people to do this properly, we need to get better at quantifying harms and benefits.

Contributors: $\mathrm{AVdB}, \mathrm{YY}, \mathrm{CJ}$, and $\mathrm{PH}$ designed the study. AVdB, CJ, and $\mathrm{JO}$ performed the analyses. $\mathrm{AVdB}, \mathrm{CJ}, \mathrm{YY}$, and $\mathrm{PH}$ drafted the manuscript. $\mathrm{AVdB}$ is guarantor.

Funding: This study was funded by the National Institute for Health Research (NIHR) Diagnostic Evidence Co-operative Oxford. The views expressed are those of the authors and not necessarily those of the NHS, the NIHR or the Department of Health. The study sponsors had no role in the design, analyses or reporting of the study. The researchers retained complete independence in the conduct of this study.

Competing interest: All authors have completed the ICMJE uniform disclosure form at www.icmje.org/coi_disclosure.pdf and declare: no support from any organisation for the submitted work; no financial relationships with any organisations that might have an interest in the submitted work in the previous three years; no other relationships or activities that could appear to have influenced the submitted work.

Ethical approval: This study was approved granted by the University of Oxford central university research ethics committee.

Transparency declaration: The lead author and guarantor of the study (AVDB) affirms that the manuscript is an honest, accurate, and transparent account of the study being reported, no important aspects of the study have been omitted, and there are no discrepancies from the study as planned.

Data sharing: We are willing to share the data that were collected in this study, which can be made available on request to $\mathrm{AVdB}$.

Welch HG, Black WC. Overdiagnosis in cancer. J Natl Cancer Inst 2010;102:605-13.

2 Moynihan R. Science of overdiagnosis to be served up with a good dose of humility. BMJ 2013;347:f5157.

3 Burgess C, Cornelius V, Love S, Graham J, Richards M, Ramirez A. Depression and anxiety in women with early breast cancer: five year observational cohort study. BMJ 2005:330:702.

4 Brodersen J, Siersma VD. Long-term psychosocial consequences of false-positive screening mammography. Ann Fam Med 2013;11:106-15.

5 Gotzsche PC, Jorgensen KJ. Screening for breast cancer with mammography. Cochrane Database Syst Rev 2013;6:CD001877.

6 Independent UKPoBCS. The benefits and harms of breast cancer screening: an independent review. Lancet 2012;380:1778-86.

7 Vaidya JS, Wenz F, Bulsara M, Tobias JS, Joseph DA, Keshtgar M, et al. Risk-adapted targeted intraoperative radiotherapy versus whole-breast radiotherapy for breast cancer: 5 -year results for local control and overall survival from the TARGIT-A randomised trial. Lancet 2014;383:603-13.

8 Chou R, Croswell JM, Dana T, Bougatsos C, Blazina I, Fu R, et al. Screening for prostate cancer: a review of the evidence for the U.S. Preventive Services Task Force. Ann Intern Med 2011;155:762-71.

9 Hewitson P, Glasziou P, Irwig L, Towler B, Watson E. Screening for colorectal cancer using the faecal occult blood test, Hemoccult. Cochrane Database Syst Rev 2007;1:CD001216.

10 Giordano L, Rowinski M, Gaudenzi G, Segnan L. What information do breast cancer screening programmes provide to Italian women? Eur J Public Health 2005;15:66-9.

11 Gummersbach E, Piccoliori G, Zerbe CO, Altiner A, Othman C, Rose C, et al. Are women getting relevant information about mammography screening for an informed consent: a critical appraisal of information brochures used for screening invitation in Germany, Italy, Spain and France. Eur J Public Health 2010;20:409-14.

12 McPherson K. Publicity of NHS breast cancer screening programme is unfair. BMJ 2011;342:d791.

13 Baum M. Ramifications of screening for breast cancer: consent for screening. BMJ 2006;332:728

14 Waller J, Douglas E, Whitaker KL, Wardle J. Women's responses to information about overdiagnosis in the UK breast cancer screening programme: a qualitative study. BMJ Open 2013;3:e002703.

15 Hersch J, Jansen J, Barratt A, Irwig L, Houssami N, Howard K, et al. Women's views on overdiagnosis in breast cancer screening: a qualitative study. BMJ 2013;346:f158.

16 llic D, Neuberger MM, Djulbegovic M, Dahm P. Screening for prostate cancer. Cochrane Database Syst Rev 2013;1:CD004720.

17 HSCIC. National bowel cancer audit. Health and Social Care Information Centre, 2014.

18 The second all breast cancer report. National Cancer Intelligence Network, 2008.

19 Trevena LJ, Davey HM, Barratt A, Butow P, Caldwell P. A systematic review on communicating with patients about evidence. J Eval Clin Pract 2006;12:13-23.

20 Wegwarth $\mathrm{O}$, Gigerenzer G. Less is more: Overdiagnosis and overtreatment: evaluation of what physicians tell their patients about screening harms. JAMA Intern Med 2013;173:2086-7.

21 Plumb AA, Boone D, Fitzke H, Helbren E, Mallet S, Zhu S, et al. Detection of extracolonic pathologic findings with $\mathrm{CT}$ colonography: a discrete choice experiment of perceived benefits versus harms. Radiology 2014;273:144-52.

22 Perneger TV, Cullati S, Schiesari L, Charvet-Bérard A. Impact of information about risks and benefits of cancer screening on intended participation. Eur J Cancer 2010;46:2267-74

23 Smith SK, Trevena L, Barratt A, Dixon A, Nutbeam D, Simpson JM, et al. Development and preliminary evaluation of a bowel cancer screening decision aid for adults with lower literacy. Patient Educ Couns 2009;75:358-67.

24 Rasky E, Groth S. Evidence-based information on mammography screening in Austria-reality or more pie in the sky? Gesundheitswesen 2013;75:e18-22.

25 Slaytor EK, Ward JE. How risks of breast cancer and benefits of screening are communicated to women: analysis of 58 pamphlets. BMJ 1998;317:263-4.

26 Dreier M, Borutta B, Seidel G, Münch I, Kramer S, Töppich J, et al. Communicating the benefits and harms of colorectal cancer screening needed for an informed choice: a systematic evaluation of leaflets and booklets. PloS One 2014;9:e107575.

27 Gigerenzer G, Gaissmaier W, Kurz-Milcke E, Schwartz LM, Woloshin S. Helping doctors and patients make sense of health statistics. Psychol Sci Public Interest 2007;8:53-96.

Accepted: 10 February 2015

\section{Cite this as: BMJ 2015:350:h980}

This is an Open Access article distributed in accordance with the Creative Commons Attribution Non Commercial (CC BY-NC 4.0) license, which permits others to distribute, remix, adapt, build upon this work non-commercially, and license their derivative works on different terms, provided the original work is properly cited and the use is non-commercial. See: http://creativecommons.org/licenses/by-nc/4.0/. 


\section{What is already known on this topic}

Overdetection is defined as the detection of a cancerous lesion through screening that would otherwise not have caused any symptoms or early death

Overdetection is the most important adverse effect of cancer screening, leading to unnecessary tests and treatment

Little is known about how much overdetection people would find acceptable for cancer screening and whether acceptability depends on the level of benefit and perceived harms

\section{What this study adds}

Acceptability is highly variable, ranging from accepting no overdetection at all to accepting overdetection in the entire population People accepted more overdetection when they perceived a higher benefit from screening

People aged 50 or over accepted less overdetection, whereas people with a higher education accepted more

\section{Tables}

Table 1| Baseline characteristics of people surveyed for views on acceptability of overdetection in cancer screening. Figure are numbers (percentage) of respondents unless otherwise specified

\begin{tabular}{lc}
\hline Mean (range) age (years) & Data \\
\hline Age distribution (years): & $46.9(19-87)$ \\
\hline $18-24$ & $110(11)$ \\
\hline $25-49$ & $450(45)$ \\
\hline $50-64$ & $230(23)$ \\
\hline$\geq 65$ & $210(21)$ \\
\hline Women & $510(51.0)$ \\
\hline White & $906(90.6)$ \\
\hline Education to degree level or above & $432(43.2)$ \\
\hline Long term condition & $346(34.6)^{*}$ \\
\hline Cancer diagnosis & $92(9.4) \dagger$ \\
\hline Ever invited screening & $563(56.5) \ddagger$ \\
\hline Ever participated in screening & $537(53.8) \S$ \\
\hline *Data missing for one person. & \\
†Data missing for 23 people. & \\
§Data missing for four people. & \\
\hline
\end{tabular}


Table 2| Rates of acceptance of overdetection in cancer screening for each scenario and each type of screening

Breast cancer $(n=510)$ Prostate cancer $(n=490)$ Bowel cancer $(n=1000)$

Scenario $1: 10 \%$ cancer specific reduction in mortality

\begin{tabular}{lccc}
\hline Median $(95 \% \mathrm{Cl})$ & $150(120$ to 197$)$ & $126(100$ to 150$)$ & $113(101$ to 142$)$ \\
\hline Accept no overdetection at all $(\%, 95 \% \mathrm{Cl})$ & $5.1(3.4$ to 7.4$)$ & $5.5(3.7$ to 7.9$)$ & $7.4(5.6$ to 9.2$)$ \\
\hline Accept overdetection in complete population $(\%, 95 \% \mathrm{Cl})$ & $10.2(7.7$ to 13.2$)$ & $7.1(5.0$ to 9.8$)$ & $8.4(6.8$ to 10.3$)$ \\
\hline Scenario 2: $50 \%$ cancer specific reduction in mortality & & & $205(152$ to 235$)$ \\
\hline Median $(95 \% \mathrm{Cl})$ & $313(250$ to 364$)$ & $231(200$ to 250$)$ & $5.2(3.9$ to 6.8$)$ \\
\hline Accept no overdetection at all $(\%, 95 \% \mathrm{Cl})$ & $3.5(2.1$ to 5.6$)$ & $4.5(2.8$ to 6.7$)$ & $9.7(7.9$ to 11.7$)$ \\
\hline Accept overdetection in complete population $(\%, 95 \% \mathrm{Cl})$ & $13.9(11.0$ to 17.2$)$ & $9.2(6.8$ to 12.1$)$ &
\end{tabular}


Table 3| Factors associated with level of acceptance of overdetection of cancer with screening in two scenarios-scenario 1 was $10 \%$ cancer specific reduction in mortality; scenario 2 was $50 \%$ cancer specific reduction in mortality

\begin{tabular}{|c|c|c|c|c|}
\hline & \multicolumn{2}{|l|}{ Scenario 1} & \multicolumn{2}{|l|}{ Scenario 2} \\
\hline & Median (IQR) acceptability & $P$ value & Median (IQR) acceptability & P value \\
\hline \multicolumn{5}{|l|}{ Sex: } \\
\hline Women & $120(19-448)$ & \multirow[t]{2}{*}{0.656} & $231(41-549)$ & \multirow[t]{2}{*}{0.334} \\
\hline Men & $112(15-450)$ & & $199(38-534)$ & \\
\hline \multicolumn{5}{|l|}{ Age (years): } \\
\hline$\geq 50$ & $100(10-401)$ & \multirow[t]{2}{*}{$<0.001$} & $142(20-501)$ & \multirow[t]{2}{*}{0.010} \\
\hline$<50$ & $158(31-482)$ & & $242(51-548)$ & \\
\hline \multicolumn{5}{|c|}{ Previous diagnosis of cancer: } \\
\hline Yes & $151(10-547)$ & \multirow[t]{2}{*}{0.624} & $244(19-525)$ & \multirow[t]{2}{*}{0.623} \\
\hline No & $110(19-448)$ & & $200(43-542)$ & \\
\hline \multicolumn{5}{|l|}{ Chronic condition: } \\
\hline Yes & $111(19-500)$ & \multirow[t]{2}{*}{0.997} & $210(34-561)$ & \multirow[t]{2}{*}{0.886} \\
\hline No & $119(19-449)$ & & $205(48-501)$ & \\
\hline \multicolumn{5}{|l|}{ Education level: } \\
\hline Degree or above & $120(20-500)$ & \multirow[t]{2}{*}{0.404} & $249(50-551)$ & \multirow[t]{2}{*}{0.016} \\
\hline Less than degree & $112(15-447)$ & & $168(26-500)$ & \\
\hline \multicolumn{5}{|c|}{ Knowledge of overdetection: } \\
\hline Yes & $113(22-401)$ & \multirow[t]{2}{*}{0.558} & $200(50-500)$ & \multirow[t]{2}{*}{0.670} \\
\hline No & $112(17-458)$ & & $205(39-548)$ & \\
\hline \multicolumn{5}{|c|}{ Ever participated in screening: } \\
\hline Yes & $120(19-491)$ & \multirow[t]{2}{*}{0.443} & $231(40-550)$ & \multirow[t]{2}{*}{0.147} \\
\hline No & $110(19-447)$ & & $151(43-500)$ & \\
\hline
\end{tabular}




\section{Figure}
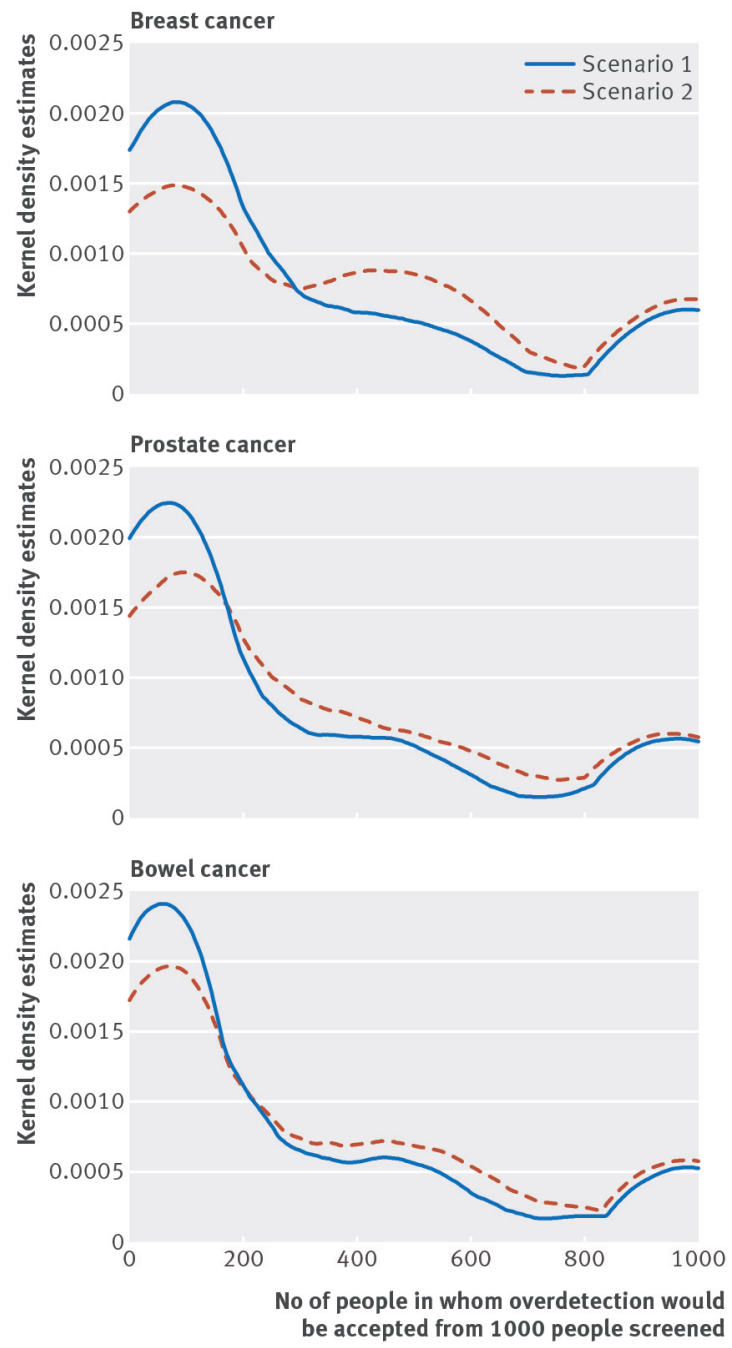

Kernel density estimates of distribution of acceptability for each scenario of each type of screening for cancer 